\title{
BEITRÄGE ZUR ENGLISCHEN GRAMMATIK.
}

\section{I.}

\section{Me. $\breve{\mathrm{a}}, \overline{\mathrm{a}}$ im Neuenglischen.}

Nach Ellis und Sweet ist das erste zeugniss uber die lautliche verschiedenheit von $\bar{a}$ und $\bar{a}$ das Butler's aus dem jahre 1633 (Ellis 64, Sweet HES.2 211). Worin diese verschiedenheit bestand, giebt uns Butler nicht an. Eine ziemlich gleichzeitige französische grammatik (Vietor, Phon. Stud. III, 188), erschienen in erster auflage 1625, in zweiter 1629 , setzt $\bar{a}=$ frz. $e$ in estre und $\breve{a}=$ frz. $a$, was wol auf $a$ (low-front) und reines $a$ (Sweet's mid-back) oder sehr nahestehende laute weist (vgl. damit das nnten angeftihrte zeugniss Erondell's).

Für diese scheidung giebt es aber noch frühere belege. Der eine findet sich in einer handschriftlich erbaltenen, wahrscheinlich aus dem jahre 1617 stammenden abhandlung Alexander Hume's 'Of the Orthographie and Congruitie of the Britan Tongue' (E.E.T.S.5). Hume war ein Schotte, hatte sich aber 16 jahre in England (Oxford, Bath) aufgehalten. In seiner abhandlung, welche die unterschiede in der aussprache namentlich des Lateinischen zwischen Nord- und Südengland darstellt, und vorschläge zu ihrer beseitigung giebt, sagt er bezüglich der aussprache des lateinischen $a$ (s. 8):

7. $A$, the first of them, the south soundes as beath thei and we sound it in bare, nudus; and we, as beath thei and we sound it in bar, obex.

9 . ... their sound of it is not far unlyke the sheepes bae, quhilk the greek symbolizes be $\eta$ not $\alpha, \beta \eta$ not $\beta \alpha$.

Also sowol in England als in Schottland waren die $a$ in bare und in bar verschieden. An die besondere lautung des $\breve{a}$ vor $r$ wie im heutigen bar ist naturlich nicht zu denken, wir haben bloss den unterschied von $\bar{a}$ und $\bar{a}$ vor uns. Welche lautwerte gemeint sind, wird nicht schwer festzustellen sein. $a$ in bare wird mit dem blöken der schafe und dem griechischen $\eta$ verglichen, welches 'as appeares be the Joneanes and Doreanes, drawes neerar to $\alpha$ than $\varepsilon^{\prime}$ (s. 10). Danach wird $\bar{a}$ den lautwert $a$ gehabt haben und dann bleibt fur $\breve{a}$ mit hinblick auf die spätere entwicklıng nichts anderes als reines $a$ oder ein demselben nahestehender laut tubrig. 
Ein noch fruheres zengniss bringt Ellis selbst in seinen nachträgen s. 226 anm., ohne seine wahre bedeutung zu erkennen. In einer französischen aussprachelehre 'The French Garden ... by Peter Erondell, London 1605' heisst es:

Our $A$ is not sounded altogether, as this english word awe as some haue written, but as the first voice of this word Augustine or After opening somewhat the mouth, as for example, Baptiste, tacitement, scauoir: and not after the rate of the english word ale, for if a Frenchman should write it according to the English sound, bee would write it in this wise $e s l$ and sound it as if there were no $s$.

Wir haben also verschiedene lautung in after und ale, d. h. (da der hentige laut in after jungen ursprungs ist) den unterschied von $\bar{a}$ und $\bar{a}$; letzteres wird dem französischen offenen $e$ gleichgestellt, was ich doch eher auf $a$ (low-front) als auf $\xi$ (midfront) beziehen möchte; ersteres dem französischen vortonigen $a$, also reinem $a$ oder höchstens dem heutigen hellen $a$ in madame. Ellis hat an ein dunkles $a$ gedacht (low-back), wol im hinblick auf das andere beispiel Augusline. Aber das au in diesem worte kann nicht den heutigen laut gehabt haben, denn es wird ja ausdricklich von awe geschieden. Es muss wol im 16. jahrhundert, als uu noch diphthong war, in vortoniger silbe das $u$ ausgefallen sein, so dass gewöhnliches $\breve{a}$ ibrig blieb. (Vgl. ubrigens anch ae. dgustinus, Agustus). Jedenfalls können wir aus der nebeneinanderstellung von Augustine und after nichts schliessen, da dem ersteren der heutige o-laut abgesprochen wird, wir also nur eine negative angabe tiber seinen laut besitzen, während die gleichstellung mit dem französischen $a$ ohne weiters klar ist.

Beide vorgefuhrten zenguisse weisen also auf $\breve{a}, \bar{a}$ oder laute, die ihnen sehr nahe stehen; eine deutliche vorstufe zu Cooper's $\breve{a,}, \ddot{c}(1685)$. Ein noch älteres zengniss fur diese scheidung bietet, wenn ich Kluge recht verstehe, die von ibm in Paul's Grundriss I, 876 angezogene französische grammatik (Rouen 1595), wonach ' $\bar{c}$ dem $e$-laut in frz. estre gleich sei'.

Die darstellung Erondell's durfte uns anch lehren, wie wir frthere, ebenfalls französische zeugnisse fur die $e$-natur des englischen "aufzufassen haben. Wenn nach dem Lambethfragment, 1528, das französische $e$ zu sprechen ist 'there proprely where the englysshe man soundeth his $a$ '(Ellis 226, 815) und 
nach Gilles du Guez (1532) 'almost as brode as ye pronounce your $a$ in englysshe' (Ellis 61), so werden sich diese angaben auf den alphabetischen lant des buchstabens, also anf $\bar{a}$ beziehen: es war naturlich, dass man bei dem zeichen $a$ zunächst an diesen laut dachte. Das 'almost' du Guez' macht wieder die lautung $c e$ wahrscheinlicher als $\ell$.

Damit stehen diese angaben zwar noch immer im widerspruch mit denen Palsgrave's, Salesbury's, Hart's und anderer, welche für $\breve{a}$ und $\vec{a}$ nur einen laut kennen und zwar nur reines $a$ oder sebr nahestehendes; aber man wird uberhaupt nicht alle zeugnisse uber $a$ unter einen hut bringen können, wie das auch Kluge Grundriss I, 876 versucht. Wie im 16. jahrhundert die erwähnten angaben, so stehen im 17. jahrhundert einerseits Erondell, Hume $(\breve{a}, \bar{a})$ und Cooper $(\breve{e}, \vec{e})$ und andrerseits Wallis $(\breve{e}, \bar{a})$ einander gegentiber, und da wir Wallis als so genauen und originellen beobachter kennen, so werden wir kaum annehmen dürfen, dass er sich geirrt hat. Ihm schliessen sich im 18. jahrbundert Ludwig (Löwisch s. 28) und Lediard (Ellis 1040) an, obwol die zuverlässigkeit dieser angaben angezweifelt werden kann. Ich glaube, wir haben hier nicht jene auseinandersetzung zwischen einem alten und einem neuen laut vor uns, wie sie bei lautwandlungen gewöhnlich sind, sondern es liegen zwei verschiedene entwicklungen vor. Die eine wird dargestellt von Palsgrave, Salesbury, Smith, Hart im 16. Jahrhundert, Gill, Hodges (Ellis 1023) und Wallis im 17., also allen jenen, bei welchen $\bar{a}$ und $\bar{a}$ denselben laut hat, die andere vom Lambethfragment, du Guez, Kluge's grammatik, Erondell, Hume, Butler, Cooper und den meisten ausländischen grammatiken ', d. h. allen, welche $\breve{a}$ und $\bar{a}$ auch lautlich scheiden. Beiden richtungen ist gemeinsam, dass das $a$ bis in den anfang des 17. jahrhunderts als reines $a$ verbleibt und dann zu $x$ ubergeht. Das $\bar{a}$ macht bei der ersten gruppe dieselbe entwicklung durch wie $\breve{a}$, bei der zweiten ist es eine stufe voraus: im 16. jahrhundert ist es schon $\bar{a}$ und geht um die mitte des 17. jalırhunderts zu $\bar{q}$ ther, wie es uns Cooper (1685)

1 Vgl. Vietor, Die Aussprache des Neuenglischen nach den deutschenglischen Grammatiken vor 1750. Marburg 1886. - Bohnhardt, Zur Lautlehre der englischen Grammatiken des 17. und 18. Jahrhunderts. Phon. Stud. II, 64, 187. - Löwisch, Zur englischen Aussprache von 1650-1750 nach frühenglischen Grammatiken. Kassul 1889. 
bietet. Diese richtung leitet anf den heutigen lautzustand hin, sie hat den sieg davongetragen.

Wie wir uns das nebeneinanderbesteben dieser beiden entwicklungen zu denken haben, wird aus der natur der zeugnisse unschwer abzuleiten sein. Die zweite ist zunächst nur durch ausländische, speciell französische zengnisse vertreten, die erste durch Engländer (und den Walliser Salesbury), und zwar gelehrte oder hofleute, die das 'beste' Englisch geben wollen, wie Palsgrave beim $a$ ansdrïcklich sagt, oder sich so grimmig gegen neuerungen wehren, wie der erzconservative Gill. Die durch sie vertretenen lautungen werden die gelehrte und höfische aussprache jener zeit darstellen, die andere dagegen die in den mittleren und niederen ständen verbreitete. Dass ausländer mit diesen ständen am ehesten und häufigsten in berührung kamen, ist begreiflich. Erst in der zweiten hälfte des 17. jahrhunderts, als durch grosse politische ereignisse die unteren schichten heraufgekommen waren, wird diese aussprache auch von einem latein schreibenden gelehrten wie Cooper dargestellt.

Anm. 1. Die deutschen grammatiken aus dem ende des 17. und anfang des 18. jahrhunderts, welche namentlich Vietor gesammelt hat (vgl. dazu Engl. Stud. 10, $361 \mathrm{ff}$ ), scheiden zunächst $\bar{a}$ wie in baker, das sie mit $\ddot{a}$ oder $a l \cdot$ wiedergeben, von dem $a$ in all, das sie dem deutschen langen $a$ gleichstellen. Fiir engl. $\breve{a}$ geben sie im allgemeinen kurzes deutsches $a$ an, fugen aber golegentlich hinzu 'doch nicht mit so vollem munde', oder es wird gesagt ' $a$ clare sonant, ut apud Latinos'; erst später wird 'kurtz a' als lautwert angegeben und auch dann findet sich daneben noch ' $k$ urtz $a$ '. Danach ist doch nicht, wie Vietor sagt, ein von dew $a$ in all nur durch quantitït verschiedener laut gemeint, sondern zunächst wol reines $a$ und später $x$. Diese grammatiker, die zeitlich sich an Cooper anschliessen, stehen also auf einem etwas veralterten standpunkt, sie geben die aussprache der ersten hälfte oder vielleicht des ersten viertels des 17 . jahrhunderts. Das ist nicht überraschend. Aehnliches gilt auch von anderen ausländischen grammatıken.

A n m. 2. Kluge sagt, Grundr. I, 876, englische grammatiker wie Smith; Hart, Bullokar, Gill unterschieden zwei verschiedene $a$-laute, einerseits far, half, afler, andrerseits name, bacon, table, makc', und constatiert dann die 'lautliche verwandtschaft zwischen $\breve{a}$ und $\bar{a}$ '. Diese bemerkung, bei der er Weymouth (0n Early Engl. Pron. 51) gefolgt zu sein scheint, ist mir nicht gan $z$ klar. Meint Kluge einen qualitativen unterschied zwischen $\breve{a}$ und $\bar{a}$ ? Dann kann ich nicht beistimmen. Gewiss finden wir in den grammatikern die angedeutete scheidung, aber sie ist nur eine trennung von kürze und länge. IIart's a, Smith's und Gill's $a$ und Bullokar's $a$ bedeuten einfach $a$ und dass dieses fiir sie denselben laut hatte wie $\breve{a}$ 
sagen Smith und Gill ausdriicklich (Ellis 63, 64), und auch von den zwei anderen haben wir genügende hinweise, dass ihr zeichen nur länge andeuten soll (vgl. Ellis 798 anm. u. 838).

\section{I, eуe, aye.}

Smith (1568) spricht in seinem werke von 'I latina, quae per se prolata, apud nos tantum valet quantum Latine, ego, ant oculus, aut etiam,...' (Ellis 112). Er meint damit offenbar die worte $I$, eye, aye, die also nach ihm vollkommen gleich lauten. Darauf beruht ja ein wortspiel in Romeo and Juliet III, 2, $45 \mathrm{ff}$. Diese gleichung nun hat Ellis zur ansicht gefuhrt, dass der laut des $\bar{i}$ im 16. jahrhundert vielfach mit den diphthongen $e i$ und ai zusammengefallen sei, obwol sie von anderen wieder geschieden wurden (122). Auch Sweet scheint (HES.2 810) in dieser stelle eine gleichstellung des $\bar{\imath}$ mit dem diphthong $a i$ zu sehen. Diese bedeutung hat nun aber Smith's bemerkung durchaus nicht.

Es muss zunächst auffallen, dass alle drei wörter noch heute denselben laut haben und zwar den laut des $\bar{\imath}$. Geht man nun der etymologie nach, so findet man, dass das vollkommen in ordnung ist. Es ist bekannt, dass eye im Mittelenglischen zwei dialektisch geschiedene, doch in London sich begegnende formen hatte, $\bar{y} e$ und eye. Erstere ist lebendig geblieben, aber die schreibung hat letztere festgehalten. Das dritte wort, aye, ist erst durch Murray im NED. aufgeklärt worden. Es ist streng zu scheiden von $a y=$ immer, aus altn. $e i$ (wovon Sweet aye $=$ ja fälschlich ableitet, HES.2 s. 316); es taucht erst um 1575 plötzlich auf und seine etymologie ist nicht klar, aber die schreibung ist in jener zeit einfach $I$ und erst im 17. jahrhundert tritt daneben ay (e) auf (zum ersten mal ey 1637 und ay 1669). Wie immer seine etymologie auch sein mag, jedenfalls haben wir den lant des $\bar{\imath}$ vor uns; die wörter $I$, eye, aye sind also nur drei verschiedene schreibungen furr genau denselben laut.

Die form $a y(e)$ durfte wol einer vermengung mit $a y=$ immer zu danken sein, die sich bei einem teil der sprechenden auch lautlich vollzogen haben muss. Cooper (1685) fuhrt 'aye pro $I$ ' unter ai-wörtern an (bait, caitiff), für welche er ausdrücklich den lautwert $a i$ angiebt (Ellis 126).

Dieselbe trias wie bei Smith erscheint nochmals bei Gill, 1621: 'Differentia significationis (quoad fieri protest, \& sonus 
permittit) orthographiâ discernitur. Sic $J$ ego, ei oculos, ëi ita'. Später sagt er von ei und $\ddot{e} i$ : 'exiguum distat ab illo qui auditur in $\delta j n$ tnus, \& mjn meus' und von $j$ 'fere est diphthongus $e i$ '. Also er scheidet und doch versichert er, dass die laute nur wenig von einander abstehen. Ich glaube, dass diese ganze scheidung eine klinstliche ist. Bei eye wäre es wol möglich, an eine erhaltung der me. form eye zu denken, welche nach Gill's gebrauch mit $e i$ wiedergegeben wäre; aber da wir sonst, so viel ich sehe, nirgends eine spur dieser form mit altem diphthong haben, so ist dies unwahrscheinlich. Und was aye betrifft, so schwankt Gill (nach Ellis 882) in seinen umschriften zwischen $e i$ und $a i$; er kennt offenbar einerseits die ursprungliche anssprache mit dem laut des $\bar{\imath}$ (damals $e i$ ) und andrerseits die analogische aussprache, die auch Cooper bezeugt, wie $a y=$ immer. Wenn er also $I$, eye, aye mit $j$, ei, $\ddot{e} i$ wiedergiebt, so bedenten alle drei bezeichnungen nichts anderes als $e i$, d. h. den damaligen laut des $\bar{\imath}$; er sucht diese wörter zu scheiden wegen der verschiedenen bedeutung und schreibung. Dies ist wichtig für die charakteristik Gill's und zeigt, dass wir bei seinen angaben vorsichtig sein mulssen.

Aus dem vorgeführten ergiebt sich auch, dass, wenn heute einige aye von eye durch einen 'breiteren', d. b. mehr ruckwärts liegenden laut unterscheiden (vgl. Murray im NED.), dies eine ebenso unberechtigte künstliche scheidung ist, wie die Gill's und einiger ausländischen grammatiken späterer zeit (vgl. Löwisch s. 49).

\section{Me. ai, ei im Neuenglischen.}

Es ist bekannt, dass bei Chaucer me. ai und ei ohne anstand im reime gebunden werden. Da Chancer ein so genauer reimer ist, der z. b. ẹu und e'u auseinander hält (Weymouth s. $104 \mathrm{ff}$.), so missen wir annehmen, dass in seiner aussprache tatsächlich die beiden laute zusammengefallen sind. Auch in der spätmittelenglischen schreibung giebt sich dieser wandel zu erkennen, indem ei und ai nicht mehr genau nach der etymologie auseinander gehalten werden, und die neuenglische schreibung hat ja dies bewahrt. Die vermischung tritt in der weise ein, dass ai vordringt und das giebt uns einen hinweis, in welchem laute sich die beiden diphthonge vereinigt haben: in ai. Da nun im heutigen Englisch ebenfalls ai und ei ver- 
schiedenster herkunft gleich lauten ', so möchte man daraus schliessen, dass nur eine fortsetzung des sprachzustandes Chaucer's vorliegt, also seit dem 14. jahrhundert die beiden diphthonge sich nicht mehr unterscheiden.

Es fällt nun sehr auf, bei den frthneuenglischen grammatikern eine scheidung zu finden. Ellis, Sweet und auch Kluge nehmen daher neben ai noch $e i$ an; ich glaube indess, dass bei näherem zusehen die sache in ein ganz anderes licht rtickt.

Palsgrave unterscheidet scharf genug zwischen beiden diphthongen, deren lautwerte er als $a+i$ und $e+i$ angiebt; aber seine beispiele zeigen, dass er ganz von der schreibung abhängig ist. Nach ihm' liegt ai auch vor in rayne und disdayne, denen doch etymologisch ei zukommt. Dass etwa das spätmittelenglische vordringen der ai für $e i$ auf einen besonderen lautwandel hinwiese, der einen teil der ei zu ai machte, ist so ziemlich ausgeschlossen; wenigstens lassen sich keine bedingungen für einen solchen lautwandel erkennen und die schreibung schwankt manchmal noch heute (gray, grey). Weiter ist bei Palsgrave bedenklich, dass er (nach Weymouth s. 47) selber zwischen payne und peyne, chayne und cheyne schwankt.

Die transcriptionen in unseren wälschen quellen, Hymn to the Virgin und Salesbury, weisen nur auf ai und unter den belegen finden sich auch solche, die etymologisch und zum teil auch in der schreibung ei haben: away, agains (HVg.) und vein (Sb.). Das einzige ei, welches $\mathrm{HVg}$. bietet, ist they und ich glaube, das ist nicht ohne bedeutung; they mag als enklitikon seinen ursprünglichen laut gewabrt halen, wie es schon einmal eine ausnahme bildete (Sweet HES. ${ }^{2}$ 705).

Wichtig ist nun das zeugniss Smith's (1568):

Inter $A i \& E i$ diphthongos minima differentia est, praesertim apud nostrates, apud nos tamen audiuntur hi soni. Fein fingere, deinti delicatus, peint pingere, feint languidus. Sed non haec tantum verba per ei pronuntiantur, sed caetera omnia per ai scripta mulierculae quaedam delicatiores, et nonnulli qui volunt isto modo videri loqui vrbaniùs per $e i$ sonant, vt haec ipsa quae nos per $e i$ scribimus, alij sonant

' Auszunehmen sind natïrlich jene ei, die schon im Mittelenglischen zu $\vec{e}$ monophthongiert waren: receive, deceive u. s. w. (vgl. Behrens in Paul's Grundriss I, 823). 
et pronuntiant per ai, tam ódı́x́ogor sumus in his duntaxat duabus diphthongis Angli.

Diese angaben widersprechen nicht denen Palsgrave's, aber sie erweitern sie: für jedes der zwei schriftzeichen bestehen also beide laute. Wol verwirft er $e i$ für das zeichen ai als geziert, doch wir wissen, was dergleichen bei einem grammatiker jener zeit zu bedeuten hat: diese aussprache war nichts anderes als eine tatsächlich bestehende oder aufkommende, die ihm entweder ungeläufig war oder nicht in sein system passte.

Dieser sprachzustand nun kann doch unmöglich eine unmittelbare fortsetzung der fruhmittelenglischen scheidung zwischen ai und $e i$ sein; er lässt sich nur so erklären, dass die beiden diphthonge zunächst zusammenfielen und dann der gemeinsame laut sich wieder spaltete, aber in anderer weise als fruher die vereinigung stattgefunden hatte. Da nun die schrift zum teil die fruhere scheidung bewahrte, kann es nicht wunder nehmen, dass die grammatiker, die ja immer von den schriftzeichen ausgingen, den wahren sachverhalt nicht erfassten, sondern meinten, den zwei verschiedenen zeichen die zwei verschiedenen laute als normale aussprache zuweisen zu mulssen. Die unbefangenen wälschen quellen dagegen geben nur einen laut, der gewiss der ursprungliche war. Woher die neue doppelheit kam, durfte sich nicht schwer ermitteln lassen. Im heu- tigen Engliseh fallen ui, ei lautlich mit $\bar{a}$ zusammen, es muss also zwischen diesen lauten eine enge beziehung bestanden haben. Ich glaube - und ich hoffe, die folgenden zeugnisse werden meine ansicht bestätigen -, dass der erste bestandteil des diphthongs genau dieselbe entwicklung durchmachte wie das $\bar{a}$, während der zweite immer schwächer wurde und endlich abfiel. Wir haben nun oben gesehen, dass sich im 16. jahrhundert fur $\bar{a}$ zwei lautungen gegentuber standen: die einen sprachen $\bar{a}$, die anderen $\bar{a}$; der diphthong musste daher bei den einen $a i$, bei den anderen ai sein. Das sind, glanbe ich, die beiden lantwerte, die Palsgrave und Smith mit ai, ei meinen.

Dabei wird man nun nicht geradezn an einen uberlangen diphthong $\bar{a} i$ zu denken halen; das $a$ im diphthoug wird in der mitte zwischen $\bar{a}$ und $\breve{a}$ gestanden haben, musste aber länger werden, je schwächer das $i$ wurde. Länge des $a$ ist uns 
ubrigens öfter angegeben und wenn Smith ausdrucklich das a als kurz bezeichnet (Ellis 121), so tut er es wol, um den gegensatz zu der gleich darauf erwähnten dialektischen aussprache $\bar{a} i$ hervortreten zu lassen und vor dieser zu warnen ( $\mathrm{vgl}$. seine worte bei Ellis 122).

Diese auffassung erklärt, wie ich glaube, ungezwungen den sachverhalt, den uns Smith erschliessen lässt, und auch die folgenden zeuguisse.

Smith macht noch weitere angaben, die die angefuhrten bestätigen. Er giebt eine reihe ai-wörter an, die zeigt, dass er in der verteilung der beiden laute wie Palsgrave von der schrift beeinflusst war; denn es finden sich darunter wai, pain, disdain, arai. Und er sagt weiter: 'qui valde delicatè voces has pronunciant, mulierculae praesertim explicant planè Romanum diphthongum ae'. Dass er hier mit 'diphthong' 'digraph' meint, kaun nicht bezweifelt werden, und der laut, den er im auge hatte, war wol $\bar{a}$, nicht $\bar{e}:$ sonst mitisste ja ai mit dem $\bar{e}$ jener zeit, geschrieben $e a$, zusammengefallen sein und im heutigen Englisch $\bar{\imath}$ ergeben haben. - Wir haben also schon so fruh monophthongische aussprache bezeugt, wenn auch nur bei den 'mulierculae'; das beweist, dass schon im 16. jahrhundert der zweite teil des diphthongs sehr schwach war.

Dies bestätigt Hart (1569), der schon durchaus monophthongische aussprache aufweist; er transcribiert ee und stellt in seinem ungedruckten manuscript $1551 a i, a y, e i, e y$ und $e a$ in eine reihe mit dem laut des $e$ in better, ever (Ellis 795, 796). Das wutrde also auf $\bar{e}$ weisen, aber aus denselben grunden wie bei Smith mutssen wir annehmen, dass ihm $\bar{e}$ vorlag. Hart sucht ja alle vocale auf die funf einfachen vocalzeichen zurtickzufuhren; den unterschied zwischen $\varepsilon$ und $a$ erfasste oder beachtete er nicht.

Hart gehört also in diesem punkte zu den fortschrittlern, welche die in mittleren und niederen schichten geltende lantung vertreten. $\mathrm{Zu}$ diesen gehören anch, wie zu erwarten, die Franzosen Erondell 1605 und Holyband 1609; sie setzen das englische $a i$ ihrem ai gleich und letzterer weist diesen laut auch dem englischen $\bar{a}$ (in gaping) zu (Ellis 227, 229): zu grunde liegt also die aussprache $\cdot a$.

Die conservativen dagegen suchen ai und ei zu scheiden. Dahin gehören Bullokar (1580), Mulcąster (1582), Gill(1621) 
und Butler (1633) und wir haben ihre bemuhung ebenso anfzufassen wie bei Palsgrave und Smith.

Bemerkenswert sind einige äusserungen Mulcaster's in seinem Elementarie: ' $A i$ is the mans diphthong, and soundeth full: $e i$, the womans, and soundeth finish in the same both sense and vse; a woman is deintie, and feinteth soon, the man fainteth not bycause he is nothing daintie'. Wir haben also hier ganz ähnliche angaben wie bei Smith, die zeigen, was wir auch sonst bestätigt finden, dass bei lautlichen varianten die frauen auf der fortschrittlichen seite stehen, d.h. mit der jungeren generation gehen. Bemerkenswert ist ferner, dass Mulcaster die verteilung von $a i$ und $e i$ (d. i. $a e i)$ nach geschlechtern fur wörter bezeugt, die auf altes $e i$ zurtickgehen, während sie Smith in dieser weise nur für ai-wörter machte. Aus seinen weiteren . bemerkungen geht tibrigens hervor, dass sein ai dem $\bar{a}$ schon sehr nahe stand.

Gill wendet sich ganz grimmig gegen Hart; er habe nicht englische wörter, sondern 'Mopsarum fictitias' dargestellt. $\mathrm{Er}$ selber transcribiert das ai der schrift mit $a i$, das $e i$ mit $e i$ : Gill ist eben erzconservativ. Uebrigens giebt er das schwanken selber zu, wenn er sagt (Ellis 114): 'Communis dialectus aliquando est ambiguus. Audies enim dai aut dei THEY, illi' Wie sehr er vom schriftbild abhängt, haben wir schon oben an seiner kunstlichen scheidung von $I$, eye, aye gesehen; werfen wir nun einen blick auf seine ei-wörter: eye, either, valleys, receive, deceive, they, their, reigneth. In eye, eilher, lag ihm offenbar der diphthong $e i$ aus me. $\bar{l}$ vor und diese stellt er in eine reihe mit den producten von me. $e i$, also $e i$ zu wi. Das zeigt deutlich, dass er die aussprache nach massgabe der schrift regulierte und seine angaben nicht wörtlich zu nebmen sind.

Butler bemerkt, dass das $a i$ 'in nachahmung des französischen' auch wie $e$ gesprochen werde.

Damit sind wir schon in's 17. jahrhundert gelangt. Wir haben oben s. 270 gesehen, dass um die mitte desselben die aussprache des $\bar{a}$ bei den einen von $\bar{a}$ zu $\bar{a}$, bei den anderen von $\bar{e} \mathrm{zu} \bar{c}$ vorritickte. Der diphthong muss daher nach unserer theorie die lautwerte $\alpha i(\bar{c})$ oder $\iota(\bar{e})$ aufweisen. Was sagt nun Wallis (1653), der in so vielen punkten neues bringt? Seine angabe tiber $a i$ ist genan so, wie wir sie erwarten sollten: 
er setzt es als $a i$ an. Bei seinen bemerkungen über $e i$ mlissen wir auf die beispiele rticksicht nehmen; die stelle ist bei Ellis nicht vollständig citiert, sie lautet:

$E i$ vel $e y$ sonatur per $e ́$ clarum seu masculinum, et $y$; vel etiam simpliciter per $\dot{e}$ clarum, suppresso sono $y$. Ut in receive recipio, seize apprehendo, deceit dolus etc. Nonnulli tamen plenius efferunt, acsi per ai scripta essent.

Die angefuhrten fälle sind lauter solche, in denen me. $\bar{e}$ (monophthongierung von $e i$ ) und $e i$ (lautwert $a i$ ) einander gegenuberstehen, eine doppelheit, die auch im Neuenglischen fortlebt (vgl. Kluge, Grundriss I, 889 anm.). Danach sollten wir bei Wallis in diesen worten erwarten: $\bar{e}$ (für me. $\bar{e}$ ) oder $a i$, daneben ęi (für me. ei); er bietet in der tat diese drei laute, nur giebt er $e i$ statt $e ̨$, wol irregefuhrt durch die lautung des monophthongs. Also auch er scheidet das ai und ei der schrift, muss aber bei letzterem zugeben, dass sie auch wie ai gesprochen werden; ganz dasselbe verhältniss wie bei früheren quellen.

Auf einem durchaus fortschrittlichem standpunkte steht Cooper 1685. Er ist der erste, welcher dem $\bar{a}$ den lautwert $\bar{e}$ zuweist, indem er es als länge dem $\breve{\varphi}$ in ken gegenuberstellt. Danach müssen wir für den diphthong den lautwert ęi oder $\bar{\varepsilon}$ erwarten. Seine angaben bestätigen uns. Die gewöhnliche lautung in der umgangssprache ist ihm $\bar{e}$, er stellt sell - sail, sent - saint u. s. w., als paare von ktirze und länge hin. Daneben kennt er noch eine diphthongische aussprache. 'Fortius prolata' habe ai in einigen wörtern den laut des diphthongs $u i$, d. h. wie aus einer anderen stelle heryorgeht, $\infty i$, nämlich in brain, frail, bait, caitiff, aye (pro I), eight; ęi liege vor in praise, height, weight, convey, hainous und anderen, in der umgangssprache werde aber in allen einfach $\bar{q}$ gesprochen. Also es gab neben seinem $\bar{e}$ auch noch $a i$ und $e i$; dieser letztere diphthong entsprach seiner entwicklungsstufe, sobald er noch einen diphthong sprach, $x i$ war fur ihn eine altertiumliche aussprache, die er aber leicht hören konnte von leuten, die der richtung Wallis' angehörten. Wenn er die wörter, in denen er diese diphthonge gelten lässt, so genau abgrenzt, so hat das keine bedeutung; es wird daher kommen, dass er selber sie kaum sprach, sondern sie nur von anderen leuten hörte und sich bei abfassung jener stelle nur an diese worte erinnerte. Uebrigens scheint auch bei ilım die scheidung zwischen ęi und aei nach 
massgabe der schriftzeichen noch einzuspielen. Das 'Fortius prolata' werden wir auf nachdrtickliches sprechen bei reden reden, auf der kanzel u. dgl. zu beziehen haben; es stimmt mit unseren sonstigen erfahrungen uberein, dass in gehobener rede die lautung wie der wortschatz altertümliche elemente bewahrt, die der umgangssprache bereits fremd sind.

Um jene zeit also, gegen ende des 17. jahrhunderts, war der diphthong ai (lautwert der schriftzeichen ai und ei) bereits mit $\bar{a}$ zusammengefallen. Von den folgenden gewährsmännern versuchen noch einige, zwischen ai und $a$ oder gar zwischen ai und $e i$ einen unterschied herauszufinden; aber es ist klar, dass das alles nur künstlich-traditionell ist. Aehnlich verhalten sich auch die ausländischen grammatikęn. Wenn vereinzelt für ai ein hellerer laut angegeben wird als fïr $\bar{a}$ (Löwisch 29), so kann dem keine bedeutung zugemessen werden. (In raisins muss tubrigens schon im Mittelenglischen monophthong gegolten haben, wie das später gelegentlich bezeugte $\bar{\imath}$ beweist, Löwisch 55.)

Die vorgetragene auffassung erklärt auch einige reime, welche Éllis 867, 872 aus Surrey, Sidney und Spenser belegt und welche Kluge, Grundriss I, 889 mit recht auffallend findet, wie claim : fame, sea: way. Die correcte, gebildete aussprache des $a i$ war fitr jene dichter gewiss noch $a i$; daneben aber gab es eine volkstumliche anssprache $a i$ mit sehr schwachem $i$, sogar schon $\bar{x}$. Diese lautung machten sie sich manchmal in reimnot zu nutze. Bindungen von $a i: a$ erklären sich also als $\bar{a}(i): \bar{e}$ (vielleicht auch $\bar{a}(\imath): \bar{a})$ und $a i: e a$ ist phonetisch $\bar{e}(i): \bar{e}$ [vgl. nachtrag].

Unser ergebniss ist also: Im Spätmittelenglischen fielen ai und ei zusammen unter ai, während die schrift noch viele ei bewahrte. Nur im enklitischen they, their scheint das alte $e i$ noch erhalten worden zu sein, während im betonten they, their ebenfalls ai eintrat (vgl. Gill). In der nenenglischen zeit macht das $a$ in ai dieselbe entwicklung durch wie $\bar{a}$, während das $i$ schwächer wird und bald ganz schwindet, so dass $a i$ und $\bar{a}$ zusammenfallen (ende des 17. jahrhunderts). Da nun fur $\bar{a}$ zwei lautungen neben einander hergingen, so entstand im diphthong dieselbe doppelheit und dies veranlasste die meisten grammatiker im 16. und 17. jahrhundert, die zwei lantungen auf die schreibungen ai und $e i$ zu beziehen und so eine ktunstliche scheidung zu machen. 


\section{Zur diphthongierung von me. $\overline{\mathbf{u}}, \mathrm{t}$.}

Ein für die englische lautgeschichte sehr wichtiges zeugniss Bullokar's (1580) lautet, nach Ellis 93:

' $O$ hath three soundes, and all of them vowels; the one sound agreeing to his olde and continued name, another sound, betweene the accustomed name of, $o$, and the old name of, $v$, and the same sound long, for which they write oo, (as I do also, but gining it a proper name, according to the sound thereof), the thirde sounde is as, $v$, flat and short, that is to say, as this sillable ou, short sounded: for which some of the better learned did many times use, oo. \&, v, according to their sounds, but most times with superfluous letters'.

Ellis fugt dann hinzu: He illustrates the three sounds by the words:

1) sonne filius, vpon, bosome (first vowel), corne, close.

2) sonne sol, out, bosome (second vowel), come.

3) loked, toke, boke, sone.

Wol jedem, der diese angaben aufmerksam gelesen hat, werden zweifel an der richtigkeit der anordnung dieser reihen aufgestiegen sein, denn sie passen nicht zu den drei lauten, welche Bullokar beschreibt. Durch die gute eines freundes, des herrn Alois Stefan, d. z. in London, bin ich im stande, den wortlaut der stelle, in welcher Bullokar seine beispiele giebt, mitzuteilen. Nach den oben angezogenen worten fährt er fort:

' $O$ of three soundes vsed in these wordes, and such like, thus: my sonne loked upon the sonne beames, and toke his boke out of his bosome as sone as I was come out of our corne close, in which writing, the first written (sonne) meaneth and signifieth him, that $I$ am father vnto: the seconde written (sonne) meaneth and signifieth the greatest light in the firmament: the thirde written (sone) meaneth and signifieth the time, when he toke the boke out of his bosome. For the which I write the first (sonne) thus: so, n', in Latine, filius; in French, fylz. The seconde thus: su,n: in Latine, sol, in French, soleil. The thirde thus: soon 2: in Latine cito: in French tost. The whole sentence I write thus: my so,n looked v,pon the su,n-bæmz', and took hiz

1 Das komma nach einem buchstaben steht im original unterhalb desselben, wie die cedille im Französischen.

\& Unser oo steht für ein $\omega$-artiges zeichen Bullokar's. 
book ou,t of hiz bozo,m, az soon az he' waz co,m ou,t of ou,r córń-clóe'.

Ellis glaubte also, die scheidung zwischen so,n, su,n und soon sei dieselbe wie frther zwischen den drei $o$-lanten; aber das ist nicht gesagt, obwol man es erwarten sollte. Wir mussen auf anderem wege herauszufinden trachten, welches seine drei $o$-laute sind. In diesen transcriptionen, welche tubrigens nur ein dtirftiger versuch sind, die herkömmliche schreibung durch diakritische zeichen den tatsäichlichen lautverhältnissen anzapassen, erscheinen folgende bezeichnungen:
a) $0:$ v,pon, of, bozo,m
b) 6: cơrn, clóe' (= close)
c) oo: looked, took, book, soon.
d) o,: so,n, bozo,m, co,m
e) $\mathfrak{u}, \mathbf{v}, \mathrm{pon}, \mathrm{su}, \mathrm{n}$
f) ou,: ou,t, ou,r.

Von diesen zeichen ist $o$ und $\dot{o}$ nur durch die quantität verschieden; denn der accent bezeichnet bei Bullokar länge (Ellis 838). Die reihen a) und b) stellen also nur einen lant dar. Bezuglich der ubrigen fälle kommt uns eine stelle auf s. 23 seines werkes zu hilfe. Er sagt da: "Here followeth in squares the vowels and diphthongs, (with sillables for the sound of diphthongs wherein is any halfe vowell,) which agree in sound'. Die folgende tabelle, neben welcher noch einmal steht: 'Vowels and diphthongs of one sounde' besteht aus zwei reihen von je acht feldern, deren jedes gleichlautende buchstaben enthält; im letzten der oberen reihe nun steht: ov, ou, $0, w 00, w \mathrm{v}, u$, 0, 00, 0o., (d. h. komma und punkt unter oo). Somit fallen die oben als d) e) f) angefuhrten reihen ebenfalls zusammen und es liegen in seinen transuriptionen nur drei laute vor:

1) upon, of, bosom, corn, close;

2) looked, took, book, soon;

3) son, bosom, come, upon, sun, out, our.

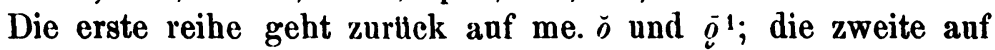
me. $\bar{o}$; die dritte anf me. $\breve{u}$ und $\bar{u}$ (geschrieben ou). Ich glaube nun, dass diese reihen, in der ordnung wie ich sie aufgezählt

${ }^{1}$ Bullokar's bosom muss in seinem wurzelvocal auf ue.bosme aus den flectierten formen von ae. hism (vgl. me.botme, fajme aus bolın-, $f a ð m$-) zurückgehen. Die verkiirzung ergiebt sich aus der mehrfachen consonanz. 
habe, in der tat auch seinen drei o-lauten entsprechen. Sein erster laut kann nicht gut anderes als $o$ gewesen sein; der gegenwärtige name des buchstaben hat denselben laut wie sonst me. $\bar{g}$ und wir haben keinen grund, an der berechtigung eines rúckschlusses zu zweifeln. Dazu passt nun allein die reihe 1): me. $\breve{o}$ und $\bar{\sigma}$ hatten ja im 16. jahrhundert nach anderen gewährsmännern den lant $\varphi$ (mid-back-wide). Bullokar's dritten lant, ' $v$ flat', bespricht er noch einmal an einer stelle, die bei Ellis s. 169 abgedruckt ist. Aus den daselbst angefuhrten beispielen geht hervor, dass er $u$ aus me. $\breve{u}$ meint und da unter den beispielen sich auch full findet, welches kaum je einen anderen laut hatte als heute und auch andere zeugnisse darauf hinweisen, so wissen wir, was er meint: den lant $u$ (high-back). Dieser lant kommt nun klärlich der reihe 3) zu, welche ja wörter mit me. $\breve{u}$ bringt. Sein zweiter o-laut, furr welchen also jetzt nichts mehr tubrig bleibt als die reihe 2) ist schwieriger zu bestimmen. Er liege, sagt er, zwischen den namen des $o$ und des $v$; da er nun den letzteren (bei Ellis 168) ausdrücklich dem frz. $u$ gleich stellt und der erstere, wie wir gesehen haben, $o$ ist, liegt also der fragliche laut zwischen $o$ und $\ddot{u}$. Das ist freilich recht unbestimmt. Der heutige laut der hierhergehörigen wörter ist $\bar{u}$ und von ihm könnte man in der tat sagen, er liege zwischen $\varphi$ und $\ddot{u}$; aber $u$ kann nicht gemeint sein, denn es liegt ja in der dritten reihe vor. Me. $\bar{g}$ kann noch nicht zu $u$ geworden sein; wir werden daher $\bar{\emptyset}$ oder ein sehr geschlossenes, dem $u$ nahestehendes $o$ als lantwert anzunehmen haben. Bullokar's ausdrucksweise scheint sonderbar; man sollte erwarten, dass er diesen laut als zwischen seinem ersten und dritten stehend bezeichnen wüde; die erklärung ergiebt sich jedoch aus seiner art, die vocale zu beurteilen. Wenn man seine verschiedenen angaben durchgeht, so findet man, dass er therall von den 'old and continued names' der buchstaben ausgeht. Da es nun für ihn im Englischen acht verschiedene vocale giebt, die vorliegen in: to lack, to leak, a leek, to lick, a lock, to look, luck, Luke (Ellis 64), so lag ihm der laut von look in der tat zwischen den namen des $o$ ( $o$ in lock) und des $u$ ( $u$ in Luke).

Wir haben also fur seine drei $o$-laute die lautwerte: 1) offenes $o$ (me. $\breve{o}, \bar{g}), 2$ ) sehr geschlossenes $o($ me. $\bar{o}), 3) u($ me. $\breve{u}, \bar{u})$ anzusetzen, dieselben, welche bereits Sweet HES. ${ }^{2}$ angenommen hat. 
Ein paar erläuternder worte bedtirfen vielleicht noch seine zwischenbemerkungen. Von seinem zweiten laut sagt er, 'they write oo' für ihn; das heisst wol, die thbliche schreibung ist oo und das trifft ja zu. Die bemerkung zu seinem dritten laut besagt meines erachtens, dass einige der 'better learned' häufig, entsprechend ihrer aussprache, für $\bar{u} \breve{u}$ in out come die zeichen oo und $v$ gebrauchten. Diese stelle enthält also einen versteckten hinweis auf die verschiedenheit der aussprache der 'better learned' und der allgemeinheit in betreff des $\bar{u}$. Wir wissen wol sonst von einem solchen orthographischen versuch nichts, aber unsere quellen sind ja im 16. jahrhundert noch so spärlich, dass wir aus diesem fehlen nichts schliessen können. Worauf der zusatz 'but most times with superflous letters' geht, ist mir freilich nicht klar.

Die wichtigkeit von Bullokar's zeugniss liegt nun darin, das me. $\bar{u}$ in seiner aussprache noch nicht diphthongiert ist, oder doch erst so schwach diphthongiert, dass er es nicht merkte, und demgemäss me. $\bar{o}$ noch nicht ganz zu $\bar{u}$ vorgerickt erscheint. Er ist um eine stufe hinter seinen zeitgenossen zurtick and stellt eine altertlimliche aussprache dar, gerade so wie Palsgrave, dem me. $\bar{u}$ und frz. ou fast gleich sind. Auch Mulcaster (1582) scheint noch monophthong gehabt zu haben (Ellis $913 \mathrm{f}$.) und Richardson, der allerdings von nordenglischer lautgebung beeinflusst zu sein scheint, zieht noch $1677 \bar{u}$ dem au vor und bezeichnet letzteres als einen sehr grossen missbrauch (Löwisch $62 \mathrm{f}$.).

Kluge bemerkt in Paul's Grundriss I, 873 anm., dass Ellis' annahme, auch Bullokar habe noch vielfach $\bar{u}$ nicht diphthongisch gesprochen, auf einer irrigen interpretation seiner orthographie bernhe und beruft sich anf Weymouth s. 30 . Nach den ausgefuhrten ist Ellis' annahme vollkommen gerechtfertigt. Uebrigens enthält Weymouth's doch herzlich unzuverlässiges buch, welches Kluge tiber gebtihr benutzt und citiert, an der betreffenden stelle nichts als den hinweis, dass Bullokar inlo, slool u. dgl. von how, ground in seiner orthographie scheide; die stellen, in welchen er so ausftihrlich uber den laut spricht, hat also Weymouth gar nicht beriicksichtigt.

Ganz parallel liegen ja die verhältnisse bei $\bar{\imath}$. Auch hier haben noch Palsgrave und Bullokar $i$-lautung und daher fur me. $\bar{q}$ ein sehr geschlossenes, dem $i$ nahestehendes $e$ (vgl. fur 
letzteres Sweet 818). Kluge läugnet dies für Palsgrave (Bullokar erwähnt er nicht) mit hinweis auf Weymouth s. 14. Aber auch hier hat sich dieser die vorliegenden zeugnisse nicht ordentlich angesehen; er teilt sie verkurzt mit und dabei sind gerade jene stellen, die gegen seine anschauungen sprechen, ansgefallen. Da das bush in Dertschland selten ist, wird es gut sein, seinen wert durch diese probe zu beleuchten. 'He states', sagt er von Palsgrave, 'that the French $i$ has two diverse sounds, one of them like the Italian $i$ and as we sound $e$ in bee, an insect, fee a reward'; while as to the second he says .... Diese stelle lautet nun in wirklichkeit (Ellis 109):

$I$ in the frenche tong hath ii. dyuerse maners of soundynges, the sounding of $i$, whiche is most generally vsed in the frenche tong, is like as the Italians sounde $i$, and suche with vs as sounde the latin tong aright, whiche is almost as we sounde $e$ in these words $a$ bee a flie, a beere for a deed corps, a peere a telowe, a fee a rewarde, a little more soundynge towards $i$, as we sound $i$ with vs. Nimmt man dazu die stelle bei Ellis s. 77, wo vom engl. $e$ in $b e e$, we u. dgl. gesagt wird, es sei 'allmoste' frz. $i$ (während sonst bei ihm solche einschränkungen nicht vorkommen), ferner die stellen bei Ellis 109 u. 110, welche engl. $y$ in by, spye, fly, awry an- oder auslautendem frz. $i(y)$, ferner engl. $w y$ in swyne, dwyne, frz. $v y$ in condvyre gleichstellen, so wird man den folgerungen Sweet's (HES.2 814 f.) beipflichten mtissen, dass Palsgrave für me. $\bar{\imath} \bar{e}$ noch $\bar{\imath}$ (oder wenig verschiedenes) und sehr geschlossenes $\bar{e}$ sprach. Ganz gleich werden frz. und engl. $i$ nicht gewesen sein, sonst whrde er an der angezogenen stelle bei frz. $i$ nicht zunächst $e$ in bee zum vergleich herangezogen haben. Es war wol zweigipflig oder schon schwach diphthongisch.

Seine angabe, dass frz. $i$ im an- und auslaut einen anderen laut habe als sonst, denselben wie in engl. by, spye u. s. w., ist recht sonderbar; zur richtigen auffassung leitet uns, glaube ich, seine beinerkung, dass frz. $i$ an diesen stellen in sorgfältig gedruckten bucheru als $y$ erscheine, sowie der umstand, dass die englischen beispiele alle $\bar{i}$ im auslaut haben und daher mit $y(e)$ geschrieben sind: er glaubte, $y$ sei vom $i$ verschieden und wollte eine lautliche scheidung dort heraushören, wo nur eine orthographische variante vorlag. 
Palsgrave und Bullokar stellen uns also die letzten ausläufer einer bereits verdrängten lautung dar; sie scheint auch noch einigen reimen, die Kluge Grundriss I, 873 anfuhrt, zu grunde zu liegen.

Andererseits haben wir, wie zu erwarten, anzeichen daftir, dass schon im Spätmittelenglischen die diplithongierung vorhanden war. Das haben namentlich Sarrazin (Lit.-Bl. V, 270) und Münster (Untersuchungen zu Thomas Chestre's Launfal. Kiel 1886, s. 29) gezeigt. Holthaus hat sogar im auftreten der schreibung ou furr ae. $\dot{u}$ bereits einen hinweis auf diphthongische lautung sehen wollen (Anglia VIII, anz. $106 \mathrm{ff}$.), während man bisher in dieser schreibung französischen einfluss erblickte. So weit düren wir aber schwerlich zurluckgehen. Das einzige ernst zu nehmende argument, welches er beibringt, ist (s.112), dass die Engländer doch vor allem von den Normannen beeinflusst wurden, bei diesen aber für frz. $\bar{o}$ (später ou) $u$ geschrieben wurde. Dagegen ist nun Sweet, HES. ${ }^{2} 590 \mathrm{zu}$ vergleicheu, der darauf hinweist, dass der französische einfluss im anfang allerdings zunächst rein normannisch war, von der mitte des 12. jahrhunderts aber, als das haus Anjou auf den tron kam und namentlich vom anfang des 13. jahrhunderts, als die staatsrechtliche verbindung mit der Normandie aufhörte, centralfranzösischer einfluss herrschend wurde. In der tat tauchen auch un jene zeit die ersten ou auf. Häufiger werden sie erst zu ende des 13. jahrhunderts. Um genau dieselbe zeit und in derselben weise tritt aber anch im Anglonormannischen ou fitr älteres $u$, ein (Sturmfels, Anglia IX, 565). Wir haben also offenbar sowol im Anglonormannischen als im Englischen nur eine orthographische änderung unter centralfranzösischem einfluss vorliegren, keinen lautwandel.

Aber im anfang des 15. jahrhunderts muss die diphthongierung schon begonnen haben. Das ist namentlich beim $\bar{\imath}$ klar. Beim $\ddot{u}$ ist die sache schwieriger, da die schreibung. nichts an die hand giebt und die reime mit diphthongischem on, on nicht immer ganz sicher sind; der me. diphthong ou ist ja zum teil (wie auch die heutige lautung beweist) fruhzeitig zu $\bar{u}$ ullergegangen (vgl. ten Brink, Chaucer $\S 33 \varepsilon$ ) und die grenzen dieses uiberganges scheinen in den einzelnen dialekten sehr verschieden gewesen zu sein. Bei den von Munster a. a. o. angefuhrten belegen von $o u: \bar{u}$ fallt z. b. auf, dass unter 
den ou-wörtern so häufig four erscheint. Das legt den verdacht nahe, ob nicht etwa in diesem worte auch $\bar{u}$ bestanden habe (neben gewöhnlichem ou) und in der tat finden wir fourth bei Hart (Ellis 112) und Price (Ellis. 157) unter wörtern, die auf me. $\bar{u}$ zurtickgehen. Ebenso ist pour bei Hart a. a. o. und Butler (Ellis 117) mit dem auf me. $\bar{u}$ beruhenden diphthong bezeugt, während seine heutige lautung me. ou voraussetzt. Diese ganze frage bedarf also noch sehr einer kritischen untersuchung. Zugleich aber wird dies beispiel zeigen, wie nötig es ist, die oft so unklaren hinweise, die uns das Mittelenglische an die hand giebt, durch die zeugnisse aus fruhneuenglischer zeit zu ergänzen. ${ }^{1}$

WIEN, am 14. Februar 1891.

KARL LUICK.

Nachtrag (zu s. 279). Vereinzelte verweclsslungen von $a$, $a i$ und $e a$ in der fruhneuenglischen orthographie, wie sie Sopp (Anglia XII, 281, 283) fur Tyndale und Lummert und Wurzner fur Shakespeare nachgewiesen haben (L., Die Orthographie der ersten Folio-Ausgabe der Sh.'schen Dramen, 1883, s. 3, 7; W., Die Orthographie der ersten Quarto-Ausgabe von Sh.'s 'Venus and Adonis' und 'Lucrece', 1887, s. 4,5), vermögen unsere obigen darlegungen nicht $\mathrm{zu}$ erschuttern. Zum teil werden sie sich ebenso erklären wie die reime von $\bar{a}: a i$, zum teil bestehen wirklich doppelformen (master, plaster) und endlich wird manches bloss druckfehler sein. Das auftreten von hair fur me. hẹr bedarf noch der aufhellung. Die erklürung Lummert's scheint mir nicht zutreffend. Die lautliche entwicklung hätte ja ibre parallele in to bear aus bẹre n. dgl. Dass aber die schreibung ai so frth auftritt, muss doch wol auf einer nebenform beruhen. Liegt etwa schottischer einfluss vor? - Ganz vereinzeltes $e$ für etymologisches $\breve{a}$ bei Tyndale (Sopp 280) kann für die qualität des $\breve{a}$ nichts beweisen.

GRAZ, am 25. Juni 1891.

KARL LUICK.

${ }^{1}$ Fortsetzung [5. Zur entstehung des ne. iu. 6. Frz. eu, ieu im Englischen. 7 . Der lautwert von me. ne. oi] folgt im nächsten heft. 


\section{BEITRÄGE ZIJR ENGLISCHEN GRAMMATIK.}

I.

5. Zur entstehung des ne. iu.

Das heutige in in new, den, duke ist, zum teil wenigstens, aus fallenden diphthongen entstanden, es muss also einmal ein umschlag von fallendem zu steigendem diphthong stattgefunden haben. Die frage: wann und warum dies geschehen ist, wurde bis jetzt noch wenig behandelt. Ellis berthrt sie gar nicht. Sweet constatiert das vorhandensein des steigenden diphthongs im 18. jahrhundert (HES. ${ }^{2} \S 881$ ). Holthaus spricht ausfilhrlicher daruber Anglia VIII, anz. 103; er findet diese wandlung sehr auffallig gegentuber dem, im Englischen doch gewahrten germanischen betonungsprincip und glaubt, ihre ursache liege in der änderung der betonung, welche die dem Französischen entlehnten wörter im Englischen erlitten. Aehnlich spricht sich Belurens in Paul's Grundriss I, 821 aus: wann und auf welchem wege der neuenglische lant sich herausgebildet habe, sei ein noch ungelöstes problem; am fruhesten durfte dies vor vocalen und in wortauslaut der fall gewesen sein, uberall aber der $j u$-laut in eine zeit zurtuckdatieren, in welcher der accent seine spätere stelle noch nicht definitiv behauptete.

Ich bin anderer ansicht. Ich glaube, die ursache dieser erscheinung - die thbrigens noch in anderen germanischen sprachen, z. b. im Altnordischen vorkommt - liegt in der natur des diphthongs $i u$, in verhältnissen, die ich an anderem orte (PBB. XVI, 336) klarzulegen gesucht habe. iu mit dem accent auf dem ersten element ist ein unechter diphthong (nach Sievers' bezeichnung), d. h. einer, dessen betoute componente von geringerer naturlicher schallfulle (schallstärke) ist als seine unbetonte. Solche diphthonge sind nach meinen oben angezogenen ausfuhrungen nicht möglich in unbetonter silbe und in betonter 
nur dann, wenn der atemdruck im verlauf der ersten componente gentigend herabgesetzt, d. h. gewöhnlich nur, wenn diese mit schwach geschnittenem accent gesprochen wird. Wir haben nun alle ursache anzunehmen, dass im Englischen seit der längung der kürzen in offener silbe, also seit der mitte des 13. jahrhunderts, kurze vocale und daher auch kurze erste componenten in diphthongen mit stark geschnittenem accent gesprochen wurden, wie noch heute: unter dem acut, mindestens unter stark ausgeprägtem, ist aber nur in möglich.

Die frage, wann iu entstanden ist, fuhhrt uns auf die rorgeschichte desselben, tuber welche schon viel gestritten wurde. Der heutige laut hat drei quellen: me. $q u$, equ und das $\bar{u}$ in französischen lehnwörtern. eu ist noch ein echter diphthong, daher konnte er sich als fallender behaupten. Wie aber $e \mathbf{z u}$ $i$ vorrtickte, die erste componente also an schallfulle verlor, musste der umschlag zum steigenden diphthong eintreten. Dies fand bei $q u$ am ende des 17. jahrhunderts statt (gleichzeitig mit dem ubergang von $\bar{e}$ aus me. $\bar{e}$ zu $\bar{\imath}$ ). Viel verwickelter ist die geschichte des $e u$, auf die wir näher eingehen müssen, zumal dabei auch die dritte quelle des heutigen $i u$, frz. $\bar{u}$ zur besprechung gelangt.

Me. $\varrho u$ und $\ddot{u}$ sind an der scheide des 14. und 15. jahrhunderts zusammengefallen, wie reime und schreibung beweisen, (nicht erst im 16. jahrhundert, wie Kluge Grdr. I, 886 sagt). Wenn nur für auslautendes oder vor vocal stehendes $\ddot{u}$ die schreibung en erscheint, so kommt dies daher, dass nur in dieser stellung die bindung des $\ddot{u}$ und $e u$ im reime stattfand: in anderen stellungen war sie nicht möglich, weil ęu nur im auslaut, vor vocal und in wenigen wörtern auch vor th sich entwickelt hatte. Ich glaube daher gegentuber Sweet (HES. ${ }^{2}$ 861) und anderen, der zusammenfall war ein vollständiger und allgemeiner. Unter welchem laut ẹu und $\ddot{u}$ sich geeinigt haben, ist aus dem Mittelenglischen schwer zu erkennen, da mussen die neuenglischen zeugnisse helfen.

Von diesen weisen nun Palsgrave 1530, Cheke 1555, Smith 1568, Hart 1569, Bullokar 1580, Cotgrave 1611, Gill 1621, G. du Grès 1636 (Vietor, Phon. Stud. III, 190), Wallis 1653 und mehrere ausländische grammatiker aus dem ende des 17. jahrhunderts (vgl. Vietor 13, Löwisch $66 \mathrm{ff}$ ) auf $\ddot{u}$. Noch Steele 1775 kennt den franz. $\ddot{u}$-laut in einigen wortern 'in the more 
refined tone of the court' (Ellis 1058) und er findet sich noch heute in einigen mundarten, ganz abgesehen vom Schottischen. Beweisend sind namentlich die zeugnisse des Franzosen du Grès und der deutschen grammatiker, welche den laut französischem $u$ und deutschem $\ddot{u}$ gleichstellen und die angaben Wallis', welcher frz.-engl. $u$ nicht nur unter den einfachen lauten anfuhrt (wäbrend er z. b. die diphthongische natur des $\bar{i}$ in ride richtig erkennt), sondern auch ausdriicklich auf die monophthongische natur des lantes im gegensatz zum diphthong in hinweist. Wenn er an einer anderen stelle verrät, dass 'einige' (quidam) auch $i u$ in wörtern sprächen, für die er früher $\ddot{u}$ angegeben hat, so ist das kein widerspruch, wie Holthans Anglia VIII, anz. 94 behauptet (vgl. Sweet HES. ${ }^{2} 878$ ).

Neben diesen zengnissen für $\ddot{u}$ haben wir andere für einen diphthongisehen laut. Eine besondere gruppe bilden unsere wälschen quellen, die Hymn to the Virgin und Salesbury, welche ubereinstimmend un transcribieren. Nach den angaben Salesbury über das wälsche $u$ kann nichts anderes gemeint sein als $\ddot{u} u$. Hätten sie $i u$ im sinne, so würden sie iw dafür geschrieben haben. - Einige französische und niederländische zeugnisse aus der zweiten hälfte des 17. jahrhunderts weisen auf $i \ddot{u}$ (Löwisch $66 \mathrm{ff}$.). - Endlich haben wir zeugnisse für $i u$. Nach Sweet ist das erste sichere das Holyband's, 1609. Erondell, 1605, ist nicht ganz klar, meint aber wol dasselbe. Indess wir haben noch fruhere belege. Wie Ellis s. 838 bemerkt, existierte schon im jahre 1566 eine ausgahe des 'French Littleton' von Holyband (der eigentlich Desainliens hiess) und bereits in dieser ausgabe findet sich jene stelle, wo er den englischen laut mit you wiedergiebt. Und ein noch älteres, wenn auch nicht so sicheres zeugniss haben wir im Lambethfragment uber französische aussprache aus dem jahre 1528 (Ellis 815). Es beisst hier: " $v$ [ought to be pronounced] in puttynge a lytell of wynde out of the mouthe thus, ou, and not you'. Das ou in dieser stelle, welches auch im alphabet als transcription des $v(=u)$ erscheint, ist rätselhaft; aber der negative teil 'not you' dieses von einem Franzosen für Engländer geschriebenen stlickes kann doch nichts anderes bedeuten, als die Engländer vor der ihnen geläufigen lautung iu zu warnen.

Wir haben also zwei hauptgruppen von zeugnissen, die eine für $\ddot{u}$, die andere furr $i u$. An eine scheidung der zwei lante 
nach der etymologie ist nicht zu denken, denn alle gewährsmänner, die sich ausfuhbrlicher aussprechen, bezeugen für wörter wie nen und duke denselben laut. Diesen angaben gegentuber wird nichts ubrig bleiben, als eine doppelheit der lantung anzunehmen, wie Kluge Grdr. I, 866 getan hat. Wären die zeugnisse für iu merklich später, so würde man geneigt sein, darin vorgeschrittenere entwicklung $\mathrm{zu}$ sehen und entstehung des $i u$ aus $\ddot{u}$ anzunehmen. Aber schon in der ersten hälfte des 16. jahrhunderts sind beide laute gesichert, während erst um 1400 equ und $\ddot{u}$ zusammengefallen sind. Ich glaube, es wird doch ein zusammenhang dieses $i u$ mit dem $e q u$ anzunehmen sein und denke mir die entwickelung so.

Me. ẹu rïckte um 1400, gleichzeitig mit der entwicklung des $\bar{e} \mathbf{z u} \bar{\imath}, \mathbf{z u} i u$ vor. Dieses $i u$ und das $\ddot{i}$ der aus dem Französischen entlehnten wörter standen sich sehr nahe, da der diphthong dieselben articulationen nach einander erforderte, welche gleichzeitig ausgefuhrt $\ddot{u}$ ergeben; und nun trat eine doppelte entwicklung ein. Einerseits wurde die gleichzeitigkeit in ein nacheinander aufgelöst, d. h. für $\ddot{u}$ trat $i u$ ein; andrerseits aber kam die gleichzeitigkeit ausschliesslich zur herrschaft, d. b. iu wurde zu $\ddot{u}$. Laute, die frther etymologisch geschieden waren, wurden jetzt varianten eines lautes. Ein mittelding zwischen beiden ist das üu der wälschen quellen, das aber wol bald verschwunden sein muss, da wir sonst keine spur davon erhalten haben; vielleicht war es eine besonderheit der wälschen aussprache des Englischen. Auch das spärlich und nur von ausländern bezeugte $i \ddot{u}$ wird nur eine art vermittlungslaut gewesen sein, wenn er uberhaupt bestand. Wie wir uns die verteilung der beiden lautungen zu denken haben, ist nach den zeugnissen unschwer zu erschliessen. Dieselben quellen, welche uns furr $\bar{a}$ und $a i$ die fortschrittliche aussprache angegeben haben, lehren auch $i u$; der diphthong war also offenbar in mittleren und niederen schichten (mit denen die ausländer zunächst in beruhrung kamen) verbreitet, während $\ddot{u}$ in der gelehrten und höfischen gesellschaft galt. Erst in der zweiten hälfte des 17 . jahrhunderts wird $i u$, wie die volkstumlichen lautungen von $\bar{a}$ und $a i$, herrschend (Cooper).

Diese auffassung erklärt reime zwischen ęu- und ęu-wörtern, die sich bei Sidney, Heywood, Spenser u. s. w. finden (Weymouth 108). Sie sprachen gewiss in sorgfaltiger rede eu 
und $\bar{u}$; aber zum zweck des reimes mochten sie das volkstumliche $i u$ ftir $\ddot{u}$ heranziehen; $e u: i u$ ist noch immer ein ungenauer reim, doch kann man denselben ihnen schon zumuten, während $e u: \ddot{u}$ selbst für sie zu stark wäre. Diese auffassung macht ferner die reime von $e u$, $e u$ und $\ddot{u}$ aus spätmittelenglischen denkmälern verständlich, die Holthaus a.a. $0.99 \mathrm{ff}$. beigebracht hat. Endlich ergiebt sich aus ihr, meines erachtens, eine befriedigende erklärung des $\ddot{u}$ für me. $\bar{u}$ und ne. $\bar{u}$ (aus me. $\bar{o}$ ) uach palatalen, welches in youth, choose von einigen gewährsmännern bezeugt ist. Beim tibergang der palatalis zum $u$ stellt sich ein $i$-glide ein, der zusammen mit dem $u$ einem $i u$ sehr nahe kommt. Da nun sonst $i u$ und $\ddot{u}$ als varianten desselben lautes galten, so konnte durch analogie auch in diesen worten sich $\ddot{u}$ einstellen.

Kehren wir nun zu der frage zurtuck, die unseren ausgangspunkt bildete: Wann entstand $i u$ ? Die antwort ist nicht mehr schwer; ungefähr um 1400, als equ zu iu vorrtickte und dieser laut auch für $\bar{u}$ eintrat. Somit fällt die entstehung des steigenden diphthongs allerdings in die zeit, wo noch die betonung romanischer wörter schwankte, aber sie hat damit nichts zu tun. Nur eine art in $u$ ist jüger: das aus me.ęu; es ist zu ende des 17. jahrhunderts entstanden.

A nm. 1. Ich vermute, dass das rätselhafte ou des Lambethfragments ein schreib- oder lesefehler für eu ist, d.h. ew. Da die mehrzahl der ew zu den $e u$ gehört, welche damals wie $\ddot{u}$ gesprochen wurden, so konnte der verfasser des fragmentes das frz. $u$ mit dem engl. en umschreiben; dass er dieses mit eu wiedergab, kann nicht wunder nehmen; auch Palsgrave fasst $e v(=e u)$ und $e n$ als gleichwertige zeichen auf (Ellis 13i) und umschreibt frz. imbue, humble, un: imbévo, évmble, evn (Ellis 189). Da nun aber neben $\bar{u}$ auch $i u$ fiir das schriftzeichen en gesprochen wurde, so wollte der verfasser des fragments vor dieser aussprache warnen; daher jener zusatz 'not you'.

Anm. 2. Ich habe unter den grammatikern, die $\ddot{u}$ bezeugen, auch Hart angefiihrt, was vielleicht einiger erlänternder worte bedarf. Ich glaube, es geht nicht an, seine verschiedenen einander widersprechenden bemerkungen ohne weiters wörtlich zu nehmen und als gleichwertig anzufuihren. Es muss doch geschieden werden zwischen den angaben in seinem ungedruckten manuscript und dem achtzehn jahre später veröffentlichten buche. In jenem sagt er (Ellis 796), frz., engl., schott. $u$ und you lauteten gleich und giebt eine beschreibung des lautes, die wörtlich genommen auf den diphthong iu weist. Das ist also eine angabe, die höchst unwahrscheiuliches, um nicht zu sagen unmögliches, bietet. In dem gedruckten werk (Ellis 168) ist der vergleich mit you weggefallen und in 
der beschreibung des lautes sind hinzufligungen gemacht, welche zeigen, dass er nur die vereinigung der für $i$ and $u$ charakteristischen stellungen der mundorgane, eine vermischung der klänge $i$ und $u$ im sinne hatte, und welche furr jeden unbefangenen unwiderleglich beweisen, dass er den laut $\ddot{u}$ meinte. Danach kann es nicht zweifelhaft sein, welches zeugniss als seine meinung $z u$ fassen ist. Bei der abfassung der ersten schrift war er sich offenbar über die sache noch nicht ganz klar. Er transcribiert trotzdem $i u$, weil ihm jene vermischung der klänge $i$ und $u$ das wesen eines diphthongs ausmachte. Was $\mathrm{ihm}$, abgesehen von der tatsächlich zu seiner zeit bestehenden variante $i u$, die richtige auffassung erschwerte, ist leicht zu ersehen, wenn man die von ihm verfolgten zwecke ins auge fasst. Es ist ihm durchaus um die 'wahren und alten laute' der fünf vocalzeichen zu tun und durch vergleichung der verschiedenen sprachen gelingt es ihm in der tat, ihren ursprünglichen lautwert festzustellen. Diese fünf einfachen zeichen reichen, da er kleinere unterschiede wie zwischen $\alpha$ und $\varepsilon$ nicht beachtet (vgl. oben 276) für alle einfachen vocallaute aus, welche das Englische damals besass, mit ausnahme eben des $\ddot{u}$; es konnte daher kein einfacher vocal, es musste ein diphthong sein. Eine solche auftassung des wortes diphthong merkt man ja bei vielen grammatikern jener zeit. Ich verweise nur auf Hume II, 11 (E. E. T. S. 8, s. 8), der auch lehrreiche beispiele über die verwechslung von 'diphthong' und 'digraph' bietet. Man wende dagegen nicht ein, dass Hart ja das zeichen $y$ zur verfugung hatte und das griechische $v$ als einfachen laut kennen musste. An der betreffenden stelle seines manuscripts (Ellis 796) stellt er gr., lat. und d. $u$ und engl. $u$ in but, unto, further einander gleich; er sprach also gr. $v$ als $\breve{u}$, während Smith z. b. es dem frz.-engl. $\ddot{u}$ gleichstellt. - Uebrigens ist vielleicht die erste angabe Hart's doch nicht falsch, wie es auf den ersten blick scheint. Ae. éow ergiebt im Mittelenglischen (vgl. ten Brink, Chaucer s. 39) $o u, e u, y o u$, yeu; es wäre also eine nebenform $(y) e n=u$ wol möglich. Diese wäre auch zu erklären als einwirkung der palatalis, ähnlich wie in choose, youth (s. oben 291). Ich habe sonst nirgends einen beleg dafür gefunden; doch steht you bei Sidney öfters im reim auf $u$, geradeso wie choose, youth (Sturmfels, Anglia IX, 571). Hart könnte diese form trotz der schreibung you gesprochen und den vergleich mit $\ddot{ } i$ in seinem gedruckten buch nur deshalb weggelassen haben, weil die mehrzahl you $=\underline{i} u$ sprach.

\section{Frz. eu, ieu im Englischen.}

Fur die feststellung der schicksale des frz. $u$ im Englischen hat man vielfach das frz. eu (ieu) verschiedenster herkunft herangezogen. Man meinte, dass diese laute im Englischen dieselbe entwicklung durchmachten (vgl. Holthaus, Anglia VIII, anz. 104; Sturmfels, Anglia IX, 571 d.). Auch Behrens ist dieser letzteren ansicht (Paul's Grdr. I, 826). Ich glaube jedoch, dass sie den weisungen, welche die französische sprachgeschichte 
und frthenglische grammatikerzeugnisse an die hand geben, widerspricht.

Es sind mehrere schichten zu scheiden, welche auf sehr verschiedenen wegen zum heutigen $i u$-laut gelangten. Die erst in neuenglischer zeit aufgenommenen eu-wörter jedweden ursprungs wie z. b. neuter, feud, pewter fallen mit dem me. ęu zusammen, sie werden also erst am ende des 17. jahrhanderts zu $i u$, i u. Die lautung eu ist uns ausdrucklich bezeugt, auch von solchen grammatikern, die für $u$ die aussprache $\ddot{u}$ angeben. Diejenigen $e u$, welche schon ins Mittelenglische eindrangen, sind ibrer mehrzahl nach solche, welche auf lat. $\rho+u$ zurtickgehen: riwle, sew, (a)dieu, Jew, Ebrew, Andrew, Mathew, Bartholomen. Sie hatten centralfrz. ieu, mussten also normannisch, nach der gewöbnlichen entwicklung des ie, eu ergeben und im Englischen mit den heimischen ęu (knew, new, trew) zusammenfallen; mit ihnen machten sie die entwicklung zu $\ddot{u}$ (iu) durch. Erst auf diesem umwege also fielen norm. ęu und $\ddot{u}$ im Englischen zusammen. In diese klasse muss auch lien (in lieu of) gehören, welches Wallis unter $\ddot{u}$ anfthrt, obwol es, so viel ich sehe, aus dem Mittelenglischen noch nicht belegt ist. - Der $\varrho u$-klasse schliesst sich nach den zeugnissen des 17. jahrhunderts beauté an.

Schwieriger ist die frage, wie die wenigen fälle von frz. eu aus o, die sich im Mittelenglischen finden, sich entwickelt haben, ob mit equ oder ęu. Es kommen in betracht nevew und courfen. Leider findet sich die lautung dieser wörter bei den grammatikern des 16. und 17. jabrhunderts nicht angegeben. - Die schreibung courfu, die skeat und Mätzner belegen, würde auf die entwicklnng als ęu hinweisen.

Zweifelhafter etymologie ist corlewe; im 'Sir Degrevant', dessen dichter sonst $e q$ und equ nicht bindet, steht es im reim mit eq. Dagegen gehen bugle und slew, welche Holthaus als hierhergehörig anführt, auf frz. u zurttek (vgl. Skeat, Etym. Dict.). In fuell fir ailteres fouelle (*ouaille), im 16. jahrhundert fewell, liegt offenbar eine störung der lautgesetzlichen entwicklung vor, deren anlass freilich noch nicht klar ist. Ebenso bedarf noch das verhältniss von lure und frz. leure der aufklärung.

Dass dagegen vortoniges hiatus- $e+u$ wie in sëur aus recurus, -ёure aus -aturu eintach $\ddot{~}$ ergiebt, ist nur eine folge der schon im Anglonormannischen eintretenden synkopierung 
des $e$ (Sturmfels, Anglia IX, 580) und darf nicht mit der entwicklung von reule aus regula auf eine stufe gestellt werden, wie dies Sturmfels Anglia IX, 571d tut.

\section{Der lautwert von me. ne. oi.}

Man hat sich verschiedentlich bemtiht, aus der schreibung, den reimen und dergleichen den lantwert der diphthonge zu ermitteln, die sich im Mittelenglischen unter den zeichen oi oy ui uy bergen. Andrerseits haben jene, die sich mit den neuenglischen grammatikerzengnissen beschäftigten, eine deutliche scheidung der $o i$-wörter in eine $o i$ - und eine $u i$-klasse festzustellen gehabt, obne ihre gründe zu erkennen (vgl. Kluge in Paul's Grundriss I, 889). Es scheint mir, dass man die hinweise, die sich aus dem Mittelenglischen ergeben und diese zeugnisse noch nicht gehörig in ihrem zusammenhange betrachtet hat.

Der erste unserer gewährsmänner, der von einem $u i$ spricht, ist Smith (1568). Er sagt zwar, der englische diphthong oi bestlunde aus $\check{o}+i$; aber er ftugt hinzu, dieses $o$ stehe nicht viel von $u$ ab und es sei vielleicht besser $v i$ statt oi zu schreiben (Ellis $132 \mathrm{f}$.). Ich habe daher im folgenden die von ihm angefuhrten wörter unter $u i$ gestellt. Bullokar (1580) giebt uns dann eine reihe oi- und eine reihe $u i$-wörter. Ebenso Gill (1621), der jedoch in einigen fällen zwischen oi und ui schwankt. Dieselbe scheidung macht Wallis (1653), nur giebt er fur das $u$ der fruheren gewährsmänner ' $\partial$ obscurum'; das $u$ hat sich also im diphthong ebenso entwickelt wie sonst $\breve{u}$, es ist zu a (oder einem ihm nahestehenden laute) geworden, so dass ein teil der oi mit dem laute des $\bar{\imath}$ (ride) zusammenfällt. Freilich fugt Wallis einschränkend hinzu: von einigen werde auch diese letztere classe als oi gesprochen. An einer anderen stelle, die Ellis nicht erwähnt (Gram. Angl. Cap. I Sect. III, s. 69 des Oxforder druckes von 1765) sagt er:

$O i$, oy, efferuntur per $o$ apertum seu clarum sed correptum, et $y$. Ut in noise strepitus, boys pueri, toys nugae, toyl labor, oil oleum. Nonnulli tamen in quibusdam saltem vocabulis potius per $\grave{o}$ vel $\grave{u}$ obscurum efferunt, ut tòil, òil, vel tūyl, $\bar{u} y l$.

Das stimmt also tiberein mit der von Ellis (133) angezogenen 
stelle. - Cooper (1685) hat in der ui-classe noch einige $u i$, zumeist aber $\partial i$; nur $\partial i$ hat der Expert Orthographist (1704). Er ist jedoch nicht, wie es nach Ellis 135 scheinen könnte, der letzte zeuge für zwei arten des oi: sie finden sich wieder bei Lediard 1725 (Ellis 1045) und noch Kenrick 1773 (Ellis 1052) kennt 'a vicious custom' 'in common conversation', oil und boil wie isle und bile auszusprechen, und von einigen wörtern wie boil, join sagt er, sie hätten ihre wahre lautung fast verloren: es würde affectiert erscheinen, sie anders als bile, jine zu sprechen. - Heute sind einige der hierhergehörigen wörter als vulgarismen tiblich, im tibrigen ist oi allgemein geworden: gewiss, wie Sweet (HES. ${ }^{2}$ \& 854) annimmt, durch eine kunstliche, vom schriftbild ausgehende reaction.

Wir wollen uns nun die von unseren gewährsmännern bezeugten. wörter, nach der etymologie geordnet, vor augen fuhren. ${ }^{1}$ Die bei Ellis nicht immer vollständig angegebenen wortlisten ergänzt zum teil Kluge in Paul's Grundriss I, 889.

I. oi aus lat. $\bar{o}, \breve{u}+i$.

a) boil ui Bll. Cp. ai W. O. L. K. - ui, oi G. toil ${ }^{2}$ ui Sm. Bll. ai W. Cp. O. K. - ui, oi G. join $u i$ Bll. Mle. G. ai L. K.

joint ui Mle. G. ai C. L. - oi Sb. ${ }^{3}$

joiner $a i \mathrm{~L}$.

injoin $a i \mathrm{C}$.

jointure $\partial i \mathrm{~L}$.

coin ui Bll. ai O. L.

loin $a i$ O. L.

foin $u i \mathrm{G}$.

point $u i$ Sm. Bll. G. Cp. ai Cp. O. L.

appoint ui Bll. Mle. ai L.

disappoint ui $\mathrm{G}$.

anoint ui Mle. ai Cp. O. L.

ointment ai $\mathrm{Cp}^{4}{ }^{4}$

1 Abkuirzungen: Bll. Bullokar; Btl. Butler; Cp. Cooper; G. Gill; H. Hart; HVg. Hymn to the Virgin; K. Kenrick; L. Lediard; Mle. Mulcaster; O. Expert Orthographist; Pg. Palsgrave; Sb. Salesbury; Sm. Smith; W. Wallis.

${ }_{2}$ Hierher gestellt, falls es nicht aus dem Hollïnd. stamınt. Vgl. Skeat.

${ }^{3}$ Nach Ellis 894 wäre oi anch bei Bll. belegt; das ist wol ein irrtun. Wenigstens bringt es Kluge nicht in der liste Bll.'s (a. a. o. s. 889).

- Bei Kluge findet sich ointwent unter den oi-wörtern Bullokar's. Ist dies ein druckfehler für ointment? 
poison ui Bll. Cp. ai O.L.

destroy ui Bll. - oi L.

buoy ui Bll. G.

b) voice oi Bll. H. G. 0 .

moist oi G. L.

moistness oi Bll.

Troy oi L.

coif oi Bll. 0 .

II. $o i$ aus lat. $\check{o}$ vor $r$.

broil $u i$ Sm. $a i$ Cp. O. L. - $u i, o i$ G.

embroil $a i \mathrm{~L}$.

coil $u i$ Sm. $a i \mathrm{~L}$.

foil sb. $u i$ Sm. Mle. ai L. ${ }^{1}$

moil ui Cp. ai Cp. O.

oil $a i$ W. K. - oi G.

soil sb. $u i \mathrm{Sm} .-u i$, oi G. oi L..2

assoil - $o i \mathrm{HVg}$. G.

spoil $u i$ Bll. G. ai L.

III. $o i$ aus lat. $\check{o}+i$.

void oi 0 .

avoid oi Bll. G. L.

coit oi 0. - ui Sm.

oister oi L.

anoy oi Mle.

IV. $o i$ aus lat. $a u+i$.

joy oi Mle. G. L.

rejoice oi Bll. G. O. L.

noise oi Bll. O. L.

cloister oi L.

choice oi O. L.

V. oi aus franc. oi (norm. $e i$ ).

coy oi Pg. L. - ui Sm.

employ oi L.

loyal oi $\mathrm{G}$.

royal oi $\mathrm{G}$.

froise oi $\mathrm{Pg}$. $\mathrm{O}$.

- Mlc. und L. scheinen das verbum zu meinen, wenigstens steht es in der umgebung von verben: das wort wäre also unter Ia einzureihen.

${ }^{2}$ Sm. meint das substantiv; ob auch G. und L., ist nicht zu ersehen. 
poise oi $0 . \mathrm{L}$.

exploit oi O. L.

VI. Reste.

a) wahrscheinlich französisch, doch unsicherer herkunft:

turmoil $u i \mathrm{Sm}$.

joist ui Btl. (vgl. Ellis 894). ai L.

boisterous oi $\mathrm{O}$.

b) aus dem Holländischen:

toy oi Mle. W. L. - $u i \mathrm{Sm}$.

hoist $a i \mathrm{~L}$.

[foist $u i, o i, a i$ Jones]

loiter oi Bll. $\partial i \mathrm{~L}$.

c) unsicheren ursprungs:

boy oi Pg. ${ }^{1}$ Bll. Mle. W. Wilkins. L. - ui Sm. H.(?)

Wie man sieht, stimmen die neuenglischen gewährsmänner im grossen ganzen mit einander uberein. Gelegentliche abweichungen und zwar immer zu gunsten des oi zeigen Sm., G. und die wälschen transcriptionen bei Sb. und in der HVg., letztere beide mit je einem beispiel. Smith wird weniger in's gewicht fallen. Er kennt nur einen laut, der nach seinen worten zwischen oi und ui liegen musste. Seine unbestimmte ausdrucksweise sowie sein öfteres abweichen von den anderen gewährsmännern lässt jedoch vermuten, dass ihm ebenso wie diesen zwei laute vorlagen, dass er aber, vom schriftbild beeinflusst, sich dieses unterschiedes nicht bewusst wurde und einen mittellaut zu hören glaubte. Die anderen abweichungen scheinen aber doch nicht auf irrtumern zu beruhen und Wallis, der freilich sich auch öfters als vom schriftbild beeinflusst erweist, sagt zweinal, neben $\partial i$ werde von einigen $o i$ gesprochen. Es scheint also schon fith ausgleichung eingetreten zu sein.

Nicht berticksichtigt sind bei obiger zusammenstellung Price (1668) und Jones (1701). Ersterer sagt (Ellis 134): 'Oy sounds broader than oi', und giebt als beispiele für ersteres: moyst, cloy, cloysters, embroyder, employ, foyl, noysom, oyl, fur letzteres: joiner, joint, boisterous, emroides, exploit, noise, ointment, poise, quoif, void. Sein 'broader' macht $o y=o i, o i=\partial i$ wahrscheinlich, aber seine listen stimmen dazu nicht ganz, meist in der weise, lass wir ai dort finden, wo wir oi erwarten

${ }^{1}$ In der wortliste bei Ellis fiilschlich mit ui angeführt, vgl. s. 130. 
sollten. - Jones' Phonography, dieses wtiste regelbuch, giebt für boil, coil, join ui an, was ja in ordnung wäre. Dann aber sagt er, $\partial i$ bestehe neben $o i$ und $u i$ in einer reihe von fällen, zumeist ui-wörtern, aber auch in froise, poise, tortois, und ai gelte in Chandois, decoy, loyal, royal, voyage. Also auch bei ihm steht vielfach $\partial i$ für das zu erwartende oi. Allem anschein nach wirft er, wie Price, aussprache und schreibung durcheinander und wir werden seinen unklaren angaben nicht viel gewicht beilegen brauchen. Doch ist bemerkenswert, dass es nach Ellis (134 anm.) in der seemannssprache waidž für voyage giebt, eine form, die auch mehrere ausländische grammatiken im 18. jahrhundert angeben (Löwisch 58), und Storm (Engl. Phil. I, 293) rile für royal aus Thackeray belegt. Es möchte scheinen, als hätte sich fruh die neigung bemerkbar gemacht, die beiden klassen von oi-wörtern zu vereinigen in der weise, dass entweder sämmtliche $\mathrm{zu}$ oi oder sämmtliche zu ai wurden. Erstere entwicklung läge vor in den vorher erwähnten abweichungen zu gunsten des oi und in Spenser'schen reimen wie chyld : spoild (Ellis 869), letztere bei Price, Jones und in modernen vulgarismen. - Die ausländischen grammatiken lassen die scheidung noch erkennen, doch sind ihre hinweise vielfach dunkel und ungenau (vgl. Löwisch $78 \mathrm{f}$., Bohnhardt 189).

Von diesen abweichungen abgesehen düfen wir aber die scheidung, welche unsere gewährsmänner zwischen ui und oi machen, eine ziemlich saubere nennen. Da sie, wie ein blick auf obige zusammenstellung lehrt, offenbar mit der etymologie zusammenhängt, so muss sie auch schon im Mittelenglischen bestanden haben. Was giebt uns nun das Mittelenglische an die hand?

Die schreibung wechselt zwischen oi(oy) und ui(uy), doch ist letzteres seltner. Stets mit oi erscheinen nach Sturmfels, Anglia IX, 573 cloistre, noise, joy, choice - voice, moist, Troy, crois $^{1}$, und ferner (Behrens, Frz. Stud. V, 1, 138) jene oi, die auf lat. $\bar{e}$ zurtickgehen. Wir erkennen in diesen fällen unsere klassen IV, Ib, V, dieselben, in denen auch neuenglisch oi erscheint. Nur in III stimmt Mittel- und Neuenglisch nicht uberein; doch findet sich avoid nach Murray im N.E.D. nur mit oi geschrieben. - Die reime, welche Behrens und Sturmfels

1 Sturmfels setzt fälschlich auch boiste hierher; es findet sich mit uy und $u$, vgl. Mätzner's Wb. 
a. a. o. angeftihrt, verbinden nie wörter, welche neuenglisch verschiedenen laut haben; dagegen finden sich reime zwischen den verschieden oi-klassen sehr häufig. Ebenso sprechen die reime Chancer's wenigstens in der Ellesmere-handschrift nicht gegen die neuenglische teilung (vgl. Cromie's Rhyme-Index to Chaucer s. 163 und 210) und gelegentliche bindungen von oi und ai weisen immer nur wörter auf, die in unseren oi-klassen stehen. - Endlich ist noch eine besondere entwicklung des diphthongs zu erwähnen, welche schon im Mittelenglischen beginnt: vor gewissen consonanten ( $s$ und verwandtem) wird $i$ absorbiert: bushel, cushion, usher, crush, frush, puncheon aus boissel, coisson, huissier, croissier, froissier, poinson. Das sich ergebende $\breve{u}$ weist auf $u i$, und in der tat gehören diese fälle den $u i$-klassen an. Nur bei usher liegen die verhältnisse nicht so klar. Zwei auffassungen sind möglich, aber bei jeder ist das ne. $u$ gerechtfertigt. Liegt frz. $\ddot{i} i$ zu grunde (nach massgabe von uis oder aber aus * ustiarius), so muss das nach dem schwund des $i$ übrig bleibende vortonige $\ddot{u}$ im Englischen ebenso $u$ ergeben wie in punish aus puniss-; birgt sich aber binter der frz. ui-schreibung ein oi (lautgesetzlich aus ösliarius), so muss es in der vortonsilbe jedenfalls oi gewesen sein, das dann im Englischen zu ui wurde. - Hierher gehören wol auch mittelenglische formen wie bustes, pussund, fusoun fur boistes, poisoned, foison (vgl. Behrens 158) und bustous, bonstous fur boisl[er]ous bei Murray, N.E. D. Auch pont lony fur point loin (Behrens $157 \mathrm{f}$.) sprechen nicht dagegen: vor $n$ schrieb man ja häufig $o$ fur $u$. Alles das sind ui-wörter. Wenn diese absorption sich bei oi-wörtern findet, so muss $o$ sich ergeben; in der tat belegt Behrens a. a. o. deuor, damoseles, reiop (= rejoyep), voce, fälle, die freilich nicht ganz beweisend sind, da das $o$ in solcher umgebung steht, dass es auch $u$ bedeuten könnte. Andererseits tritt consonantierung der ersten componente zu $u$ bei $u i$, nicht aber bei oi ein: anguish und quiver (ans cŭprcum, Sturmfels, Anglia IX, 576).

Ich glaube also, dass (mit ausnahme der klasse III) im Mittelenglischen dieselbe scheidung bestand wie im Neuenglischen. Welches die beiden laute waren, ist nicht so sicher, als es auf den ersten blick scheinen möchte. Es können sich $u i$ und $o i$, aber auch $o i$ und $o i$ gegentubergestanden haben. Letzteres wurde durch die schreibung ooi, 
die sich am ende des 15. und anfang des 16. jahrhunderts nicht selten findet, nahegelegt werden: das ne. $u$, fur welches Gill länge des ersten bestandteiles angiebt, wäre aus $\bar{\partial} i$ entstanden wie ne. $\bar{u}$ aus $\bar{o}$. Gegen diese auffassung spricht einmal die weiterentwicklung des $u$ zu $\partial$, die auf $\breve{u}$ deutet, obwol allerdings im 16. jahrhundert eine verkurzung des $\bar{u} i$ zu $\breve{u} i$ eingetreten sein könnte, namentlich aber die erwägung, dass franc. $o$, agn. $u$ im diphthong aller wahrscheinlichkeit nach dieselbe lautung hatte wie als selbständiger vocal, nämlich $u$. Jene monophthongierungen durch absorption des $i$, die ja in sehr fribe zeit zurtickreichen, weisen auch auf $u$, nicht auf oi. Es wird also ebenso $u i$ und oi bestanden haben wie im Nellenglischen. Die schreibung ooi war wol ein versuch, die qualität des ersten bestandteiles auszudrilicken, ohne rilcksicht auf seine quantität. - Dass dieses $u i$ im Mittelenglischen gewöhnlich mit oi oy bezeichnet wurde, kann nicht auffallen: man tat dies der deutlichkeit halber, gerade so, wie man vor $m n u(=v)$ und nach $w$ für $u$ zumeist $o$ schrieb. 1

Die verteilung der beiden laute auf die verschiedenen klassen ist ohne weiters klar bei Ia, IV und V. In Ib sollte man der etymologie nach $u i$ erwarten wie in Ia, doch weist das Mittel- und Neuenglische ubereinstimmend auf oi. Es mlissen besondere einflusse das zu erwartende $u i$ beseitigt haben. Bei voice und moist liegt es nahe, an einen dissimilatorischen einfluss des vorausgehenden stimmhaften labialen dauerlautes zu denken; namentlich bei voice wird dies wahrscheinlich genannt werden durfen. Wann diese veränderung erfolgte, ob vielleicht schon im Anglonormannischen, bleibt zu bestimmen. Troy erklärt sich ohne weiteres als eigenname, der gelehrtem einfluss ausgesetzt war. Crois, welches häufig im Mittelenglischen mit vois reimt, wird sein $o i$ wol dem im Neuenglischen allgemein gewordenen cross zu danken haben. Coif weiss ich nicht zu erklären. Ist vielleicht die schreibung quoif, die sich öfters im 16. und 17. jahrhundert findet, doch nicht ohne lautlichen hintergrund, so dass das oi ebenso zu erklären wäre wie in vois? - In der II. klasse, lat. $\check{o}$ vor $r$,

1 Ich bekenne mich zu dieser ansicht trotz der einwände, die neuerdings gegen sie erhoben wurden, namentlich mit rïcksicht auf die friihneuenglischen zeugnisse (vgl. z. b. oben s. $280 \mathrm{ff}$.). Freilich darf man sie nicht so unvollständig citieren und willkürlich auslegen, wie dies Holthaus getan hat. 
ist ui auffällig; da aber der lautwert fur's Englische feststeht, muss seine begriindung im Normannischen liegen. Nur ein wort scheint abzuweichen: assoil ist neuenglisch nur mit oi bezeugt (allerdings nur zweimal und nicht ganz zuverlässig) und auch mittelenglisch erscheint es nach Sturmfels, Anglia IX, 576 und Murray im N.E. D. stets mit oi. Liegt in dieser klasse etwa ausgleichung zu grunde? Dagegen sprechen die substantive. - Sehr schwierig ist die erklärung der klasse III. Nach Behrens, Grundriss I, 825 gilt für $\check{o}+i$ im Norm. $\ddot{u} i$, in den verben $\ddot{i} i$ und $o i$ je nachdem stamm oder endung betont sind. In isolierten formen sollten wir danach me. $\vec{u}$, ne. $i u$ erwarten und dies findet sich auch in pew; auch die entwicklung cuii >qui, wofür Sturmfels Anglia IX, 576 einige belege bringt, ist damit vereinbar. Aber oister weicht ganz ab und wenn auch in den verben oi in vortoniger silbe berechtigt ist und von da ins Englische gedrnngen sein kann, so wäre doch, da der diphthong in vortoniger silbe zu oi werden musste, $u i$ zu erwarten. Indessen erscheint me. neben anuy, das im westen zu einfachem any wird, vielfach $g i$ (bei Chaucer im reime auf joye, Troye Troil. 4, 253) und in avoid scheint abgesehen von dem vereinzelten yvewdil Fer. 3131 nur die schreibung oi vorzukommen (vgl. Murray im N.E.D.), die freilich auch $u i$ bedenten könnte. Die grammatiker kennen in beiden nur oi. Liegt etwa in avoid (void) und coit derselbe einfluss vor wie in voice, coif?

$\mathrm{Zu}$ den resten sei noch bemerkt, dass joist fur afr. giste kaum so erklärt werden kann, wie Storm 293 und Skeat (Etym. Dict.) wollen: $o i$ sei hier für $\bar{\imath}$ (d. i. $a i$ ) eingesetzt worden zu der zeit, als die kunstliche einsetzung des oi fur $a i$ in point und ahnlichen stattfand (cbenso wie im substantiv boil fur bile). Das wort ist nämlich nach Ellis s. 894 schon bei Butler mit ui belegt. Oder denkt Butler an ein anderes wort? - Boisterous wird von Murray (N.E.D.) zu afr. boisteux gestellt, von Skeat aus dem Keltischen abgeleitet. Gegen erstere deutung spricht das freilich nur von Lediard belegte oi statt des zu erwartenden $\partial i$ (vgl. mittelenglische schreibungen wie buyste, buste bei Mätzner).

Wir können demnach als unser ergebniss hinstellen:

Fr. oi (ui) hat im Englischen zwei entsprechungen:

1) $u i$ (später $\partial i$ ) fur lat. $\bar{\sigma}, \breve{u}+i$; fur lat. $\ddot{o}+r$. 
2) $o i$ für lat. $a u+i$; für franc. oi aus älterem $e i$; für lat. $\delta$, $\breve{u}+i$ nach gewissen consonanten; für lat. $\breve{o}+i$ (neben $\bar{u}$ aus $\ddot{u} i$ ).

Eine andere ansicht tuber den lautwert von me. oi hat Sturmfels Anglia IX, 575 ff. anfgestellt; er fasst oi als ui, welches jedoch 'im allgemeinen sich im 16. jahrbundert zu oi, das noch heute gesprochen wird, verwandelte'. Die obigen ausführungen dürten die unhaltbarkeit dieser ansicht darlegen.
WIEN, am 14. Februar 1891.
KARL LUICK.

\section{ZU ALT- UND MITTELENGLISCHEN DICHTUNGEN. ${ }^{1}$}

II.

16. Die nördliche Benedictinerregel.

Einige verbesserungen zu dem in den Engl. Stud. II, 61 ff. gedruckten texte giebt bereits der herausgeber s. 384 .

S.61, v. 46. So pat pen he vonches saue. Die hs. hat sen statt pen, und dies (= ae. sidðau) war beizubehalten.

62, 92. Ryu we suld whyls we haue day. Der vers entspricht dem lat. currile, dum lumen uile habelis (in Schröer's ausgabe der Winteneyversion der reg. S. Benedicti p. 4, 5), woraus sich die besserung Ryn statt Ryu sofort ergiebt.

ib. 94. Pat mens, we suld lyf wele iwane (: tayne). Die erklärung des herausgebers : 'iwane $=$ in wane (in dürftigkeit)' ist jedenfalls unrichtig; wir haben ohne zweifel imane 'gemeinsam, zusammen' st. iwane zu lesen.

ib. 128. Die abkiirzung iћc ist nicht durch 'iesu crist' aufzulösen, wie Böddeker in der fussnote meint, soudern, was auch der reim vs verlangt, durch Jesus. Jene schreibung entspricht bekanntlich der griechischen abkürzung $I H \Sigma$.

63, $156 \mathrm{f}$. And pay pat standes and twels til end Ogans pe fendyng of pe fend,

lies dwels st. twels. Es ist eine anspielung auf Matth. 10, $22(=24,13)$ : qui autem perseveraverit usque in finem, hic salvus erit.

ib. $168 \mathrm{f}$. For thurth oure mightes mor no les May we begyn nonekyns gude,

haben keinen reim. Nach v. 186 f.: And who so makes myrth mor or min, In god behoues his ioy begin, können wir die verse leicht einrenken:

1 Vgl. Anglis XIII, 357 ff. 